\title{
Carbonic anhydrase II. A novel biomarker for gastrointestinal stromal tumors
}

Seppo Parkkila ${ }^{1,2}$, Jerzy Lasota ${ }^{3}$, Jonathan A Fletcher ${ }^{4}$, Wen-bin Ou ${ }^{4}$, Antti J Kivelä ${ }^{5}$, Kyösti Nuorva ${ }^{6}$, Anna-Kaisa Parkkila ${ }^{7}$, Jyrki Ollikainen ${ }^{8}$, William S Sly ${ }^{9}$, Abdul Waheed ${ }^{9}$, Silvia Pastorekova ${ }^{10}$, Jaromir Pastorek ${ }^{10}$, Jorma Isola ${ }^{2}$ and Markku Miettinen ${ }^{3}$

${ }^{1}$ Department of Anatomy, University of Tampere and Tampere University Hospital, Tampere, Finland; ${ }^{2}$ Institute of Medical Technology, University of Tampere and Tampere University Hospital, Tampere, Finland; ${ }^{3}$ Department of Soft Tissue Pathology, Armed Forces Institute of Pathology, Washington, DC, USA;

${ }^{4}$ Department of Pathology, Brigham and Women's Hospital, Harvard Medical School, Boston, MA, USA;

${ }^{5}$ Department of Surgery, Jyväskylä Central Hospital, Jyväskylä, Finland; ${ }^{6}$ Department of Pathology, Jyväskylä Central Hospital, Jyväskylä, Finland; ${ }^{7}$ Department of Neurology, School of Medicine, University of Tampere and Tampere University Hospital, Tampere, Finland; ${ }^{8}$ Department of Mathematics and Statistics, University of Tampere, Tampere, Finland; ${ }^{9}$ Department of Biochemistry and Molecular Biology, Saint Louis University School of Medicine, St Louis, MO, USA and ${ }^{10}$ Center of Molecular Medicine, Institute of Virology, Slovak Academy of Sciences, Bratislava, Slovak Republic

\begin{abstract}
Gastrointestinal stromal tumors (GISTs) are clinically distinct mesenchymal tumors, which generally result from expression of mutant KIT or PDGFRA receptor tyrosine kinase oncogenes. Most GISTs feature strong expression of KIT that serves as a crucial diagnostic adjunct. However, a subset of tumors lacks KIT expression and otherwise may also be difficult to distinguish from other sarcomas, including leiomyosarcoma. Because various carbonic anhydrase (CA) isozymes have been identified as potential treatment targets against different cancers, we evaluated CA II expression in 175 GISTs. Western blotting experiments indicated that CA II is highly expressed in GIST cell lines. Immunohistochemically, 95\% of GISTs showed positive signal. The CA II expression in GISTs did not correlate with particular KIT or PDGFRA mutation types. CA II immunoreactivity was absent or low in other mesenchymal tumor categories analyzed. High CA II expression was associated with a better disease-specific survival rate than low or no expression (Mantel-Cox test, $\boldsymbol{P}<\mathbf{0 . 0 0 0 1}$ ). The present results indicate that CA II is overexpressed in most GISTs, is quite selective to this tumor type among mesenchymal tumors, and therefore might be a useful biomarker in diagnostics.
\end{abstract}

Modern Pathology (2010) 23, 743-750; doi:10.1038/modpathol.2009.189; published online 15 January 2010

Keywords: biomarker; cancer; carbonic anhydrase; GIST; sarcoma

Gastrointestinal stromal tumors (GISTs) are the most common mesenchymal tumors of the gastrointestinal tract and include examples with low biologic potential and those that behave as metastasizing sarcomas. KIT expression is a nearly consistent phenotypic feature of GISTs, and oncogenic activa-

Correspondence: Professor S Parkkila, MD, PhD, Institute of Medical Technology, University of Tampere, Biokatu 6, FI-33520 Tampere, Finland.

E-mail: Seppo.Parkkila@uta.fi

Received 4 June 2009; revised 25 November 2009; accepted 7

December 2009; published online 15 January 2010 tion of KIT or PDGFRA receptor tyrosine kinase signaling is considered pathogenetically important. $^{1-3}$ GISTs are believed to originate from cells that differentiate along the interstitial cell of Cajal lineage $\mathrm{e}^{4}$ and can arise in various gastrointestinal locations. Approximately $60 \%$ of GISTs arise in the stomach, $30 \%$ in the small intestine, and $10 \%$ in other locations. ${ }^{3}$

Previously classified as smooth muscle tumors, GISTs are now considered a separate tumor entity. ${ }^{5}$ The key diagnostic feature of GIST is immunohistochemical KIT expression. ${ }^{2,6}$ However, some GISTs (no more than $5 \%$ of all cases), especially PDGFRA 
mutant gastric GISTs, may show low or undetectable KIT expression. ${ }^{7}$ This can potentially lead to an incorrect diagnosis in patients who would benefit from treatment with receptor tyrosine kinase inhibitors. $^{8}$

Several immunohistochemical markers are useful in KIT-negative GISTs, but none of them are expressed in all GISTs. CD $34,{ }^{3}$ heavy caldesmon, ${ }^{9,10}$ and nestin $^{11}$ are expressed in approximately $70 \%$ of GISTs, but they are not specific and are also expressed in other mesenchymal tumors. Most GISTs, including KIT-negative cases, express the protein kinase $\mathrm{C}$ theta, a downstream effector in the KIT signaling pathway, ${ }^{12,13}$ and a DOG1/anoctamin 1, a newly characterized chloride channel protein. ${ }^{14,15}$ Although the expression of these proteins is relatively restricted to GIST among other mesenchymal tumors, these markers have not yet been adopted widely in the routine diagnostic work-up of GIST.

As carbonic anhydrase (CA) isozymes have been reported to represent potential diagnostic and therapeutic targets in cancer, this study was undertaken to evaluate the CA expression in GISTs. These enzymes are commonly expressed in malignant tumor cells in which they promote tumor growth by contributing to intracellular alkalization and extracellular acidification. ${ }^{16}$ Our immunohistochemical screening included 175 GISTs of gastric and small intestinal origin. The results show that CA II is highly and apparently selectively expressed in GISTs establishing it as a novel biomarker for GISTs.

\section{Materials and methods}

\section{Tumor Specimens and Clinical Data}

Formalin-fixed and paraffin-embedded tumor samples were obtained from the files of Jyväskylä Central Hospital, Finland, and the Armed Forces Institute of Pathology in Washington, DC, USA, as approved by the corresponding Institutional Review
Boards. All together our tumor materials included 175 GISTs. The other tumor categories analyzed are shown in Figures $2 \mathrm{~b}$ and 4 and Table 1. Of the GISTs, $64.5 \%$ originated from the small intestine and $35.5 \%$ from the stomach. Histologically, $67 \%$ of GISTs were of spindle cell type, $15 \%$ were of epithelioid type, and 18\% showed mixed cytomorphology. Follow-up was available on 119 cases, and the median duration of follow-up was 9 years (range $<1-30$ years). The outcome categories were as follows: $34 \%$ of GIST patients died of the disease, $24 \%$ died of unrelated causes, $39 \%$ were alive with no evidence of the disease, whereas $3 \%$ were alive with the disease.

\section{Immunohistochemistry}

The polyclonal rabbit antibodies against human CA I, II, and XII have been characterized and produced previously. ${ }^{17-19}$ The monoclonal antibody M75 against human CA IX has also been described previously. ${ }^{17,18,20}$

Five-micrometer sections were processed for immunoperoxidase staining, which was performed using an automated Lab Vision Autostainer 480 (LabVision Corporation, Fremont, CA, USA). Automated immunostaining was performed using Power Vision + Poly-HRP Immunohistochemistry kit (ImmunoVision Technologies, Burlingame, CA, USA) reagents and included the following steps: (1) rinsing in wash buffer; (2) treatment in $3 \% \mathrm{H}_{2} \mathrm{O}_{2}$ in $\mathrm{ddH}_{2} \mathrm{O}$ for $5 \mathrm{~min}$ and rinsing with wash buffer; (3) blocking with cow colostrum diluted 1:2 in Tris-buffered saline containing $0.05 \%$ Tween-20 for $30 \mathrm{~min}$ and rinsing in wash buffer; (4) incubation with primary antibody (rabbit antihuman CA I, CA II, or CA XII, monoclonal M75 antibody against human CA IX) for $30 \mathrm{~min}$; (5) rinsing in wash buffer for $3 \times 5 \mathrm{~min}$; (6) incubation in poly-HRP-conjugated anti-rabbit/ mouse IgG for $30 \mathrm{~min}$ and rinsing in wash buffer for $3 \times 5 \mathrm{~min}$; (7) incubation in DAB (3,3'-diaminobenzidine tetrahydrochloride) solution (one drop of

Table 1 CA II-positive immunostaining in different tumor categories 
DAB solution A and one drop of DAB solution $\mathrm{B}$ in $1 \mathrm{ml} \mathrm{ddH_{2 }}$ O) for $6 \mathrm{~min}$; (8) $\mathrm{CuSO}_{4}$ treatment for $5 \mathrm{~min}$ to enhance the signal; and (9) rinsing with $\mathrm{ddH}_{2} \mathrm{O}$. All procedures were performed at room temperature. The mounting of the sections was performed using Entellan Neu (Merck, Darmstadt, Germany). The staining for CA isozymes was scored by three investigators (SP-Finnish study group; JL, MMUS study group) on a scale from 0 to 3 . Immunoreactivity was scored as follows: 0, no reaction; 1 , weak reaction ( $<10 \%$ positive cells); 2 , moderate reaction (10-30\% positive cells); 3 , strong reaction ( $>30 \%$ positive cells).

\section{Western Blotting}

GIST cell lines and 11 fresh-frozen samples of primary GISTs were examined. All reagents for SDS-PAGE and western blotting were from Invitrogen (Carlsbad, CA, USA) or Bio-Rad Laboratories (Richmond, CA, USA). The electrophoreses were performed under reducing conditions using NuPA$\mathrm{GE}^{\mathrm{TM}} 10 \%$ Bis-Tris gels. The proteins were transferred electrophoretically from the gel to a polyvinylidene fluoride membrane according to the standard procedures. After transblotting, CA II protein was detected by the ECL method (Amersham Biosciences, Buckinghamshire, UK) according to the manufacturer's instructions. Primary CA II antibodies were diluted 1:2000.

\section{Results}

\section{Expression of CA II in GIST}

Prompted by the observation of strong CA II expression in two GISTs (Figure 1), a total of 175 GISTs (two cohorts including 152 and 23 cases) were examined for this marker. Most GISTs (95\%) showed positive reactivity for CA II, whereas only eight cases in the former cohort were negative (Figure 2a). We also immunostained 23 GISTs with a commercial rabbit antihuman CA II antibody (Sigma, St Louis, MO, USA). This antibody generally showed a slightly weaker reactivity, but the distribution pattern was similar compared with the first antibody (data not shown). Although the peak values remained lower with the commercial antibody, probably because of a lower titer or lower affinity, the staining results showed statistically significant correlation $\left(r^{2}=0.63, P=0.001\right)$.

The present series included 134 GISTs in which KIT expression was analyzed, of which eight cases $(6.0 \%)$ were KIT-negative. Four of these eight KITnegative GISTs (50\%) expressed CA II strongly. Similarly, out of 175 GISTs immunostained for CA II, eight cases were negative, and four of these CA IInegative GISTs expressed KIT. When CA II and KIT immunostaining results were combined, only four GISTs were negative for both antigens. Four DOG1- negative gastric epithelioid GISTs were all strongly positive for CA II. Three small intestinal tumors considered GISTs that were negative for both KIT and DOG1, were also negative for CA II.

\section{CA II Expression in Other Tumors Than GISTS}

CA II expression was studied in two independent tumor cohorts. The cohort collected in Finland included GISTs, leiomyosarcomas, leiomyomas, and desmoid tumors, and the other cohort consisted of tumors from several categories collected at the Armed Forces Institute of Pathology. The immunostaining results are summarized in Table 1 and Figures 2b, 4, and 6. Although a great majority of non-GIST mesenchymal tumors were negative, sporadic CA II positivity was encountered in various tumors. There was weak, diffuse cytoplasmic positivity in some esophageal leiomyomas. Sporadic gastric schwannomas, solitary fibrous tumors, and melanomas metastatic to small intestine were variably positive. In these tumors, neovascular capillary endothelial cells, dendritic antigen presenting cells, and multinucleated histiocytes were often positive.

\section{Western Blotting}

To confirm the specificity of the CA II antiserum we subjected total proteins of GIST882 cells ${ }^{21}$ to western blotting. The rabbit antihuman CA II serum detected only a single polypeptide of $30 \mathrm{kDa}$, similar to recombinant CA II (Figure 3a). Western blotting confirmed that most GISTs express CA II, irrespective of the KIT or PDGFRA mutation type (Figure $3 \mathrm{~b}$ ). Strong CA II expression was found in 10 of 11 primary GISTs, whereas CA II expression was weak in the remaining case.

\section{Expression of Other CA Isozymes}

A subset of tumor specimens was also immunostained for the isozymes CA I, CA IX, and CA XII. These isozymes were usually either absent or only weakly expressed in GISTs and true smooth muscle tumors (Figure 4). The highest reactivity for CA IX was observed in desmoid tumors. Another cytosolic isozyme (CA I) showed low expression levels in GISTs (15 of 23 tumors were negative).

\section{Correlation of CA II Immunoreactivity of GIST with Other Tumor Parameters}

The difference in CA II expression between GISTs and leiomyosarcomas was highly significant $(P<0.0001$, unpaired $t$-test $)$. In comparing gastric $(n=54)$ vs small intestine $(n=98)$ GISTs, mean intensity score of the CA II immunoreactivity was slightly lower in gastric $(2.5 \pm 0.1$ (s.e.m.)) than in 

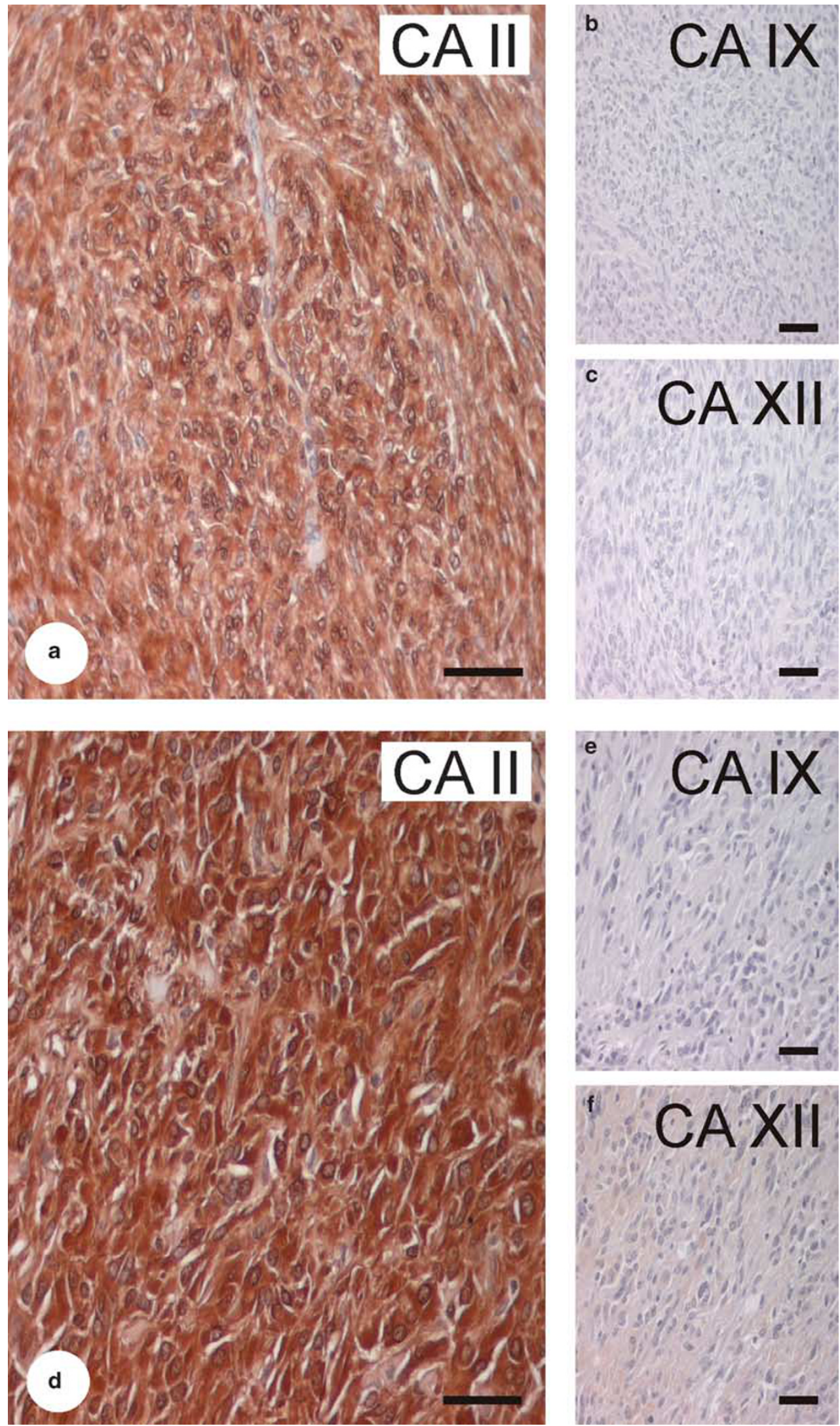

Figure 1 Two representative gastrointestinal stromal tumors (GISTs) immunostained for carbonic anhydrase II (CA II) (a,d), CA IX (b,e), and CA XII (c,f). CA II shows strong immunoreaction, whereas CA IX and XII remained negative. Bars $=50 \mu \mathrm{m}$. 

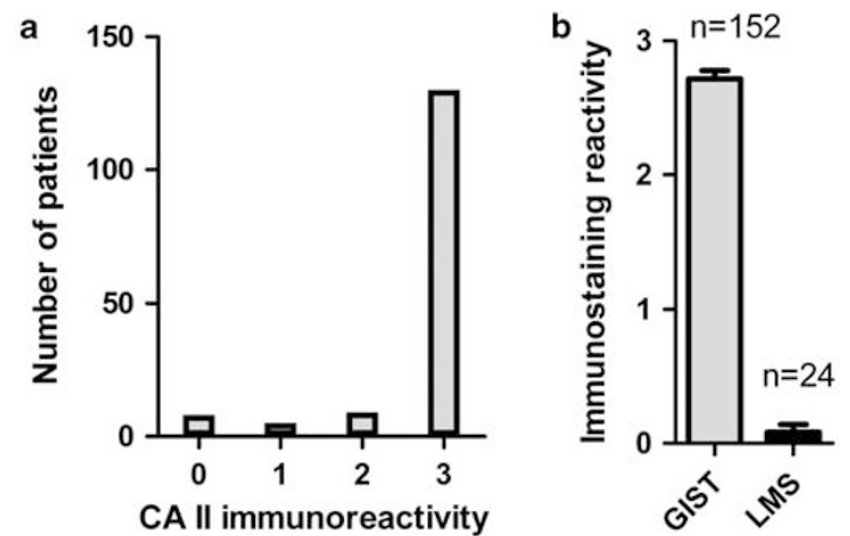

Figure 2 (a) Carbonic anhydrase II (CA II immunoreactivity in 152 gastrointestinal stromal tumors (GISTs). Most specimens showed strong signal for CA II enzyme. (b) Comparison of mean $(+/$ - s.e.m.) CA II immunoreactions in GISTs and leiomyosarcomas (LMS). CA II usually showed strong immunoreactions in GISTs, whereas LMS specimens showed negligible signals.

a

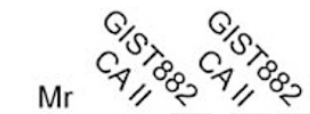

$50 \mathrm{kDa}-$

$37 \mathrm{kDa}$ -

$25 \mathrm{kDa}-$

20 kDa-

aCA II NRS

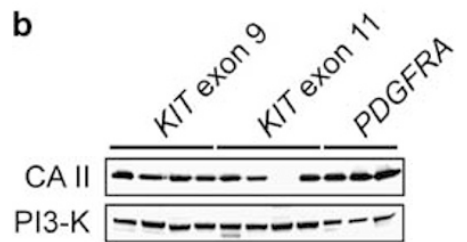

Figure 3 (a) Western blotting of carbonic anhydrase II (CA II) in gastrointestinal stromal tumor 882 (GIST882) cells. A positive $30 \mathrm{kDa}$ polypeptide of CA II was observed in the cultured cells. Recombinant human CA II was used as a positive control (the first lane). NRS = normal rabbit serum. (b) In western blotting of primary tumors, CA II was expressed strongly in most GISTs, irrespective of KIT or PDGFRA mutation type. Phosphoinositide3-kinase (PI3-K) stain was a loading control.

small intestine (2.8 \pm 0.1 (s.e.m.)) GISTs $(P=0.0091$, unpaired $t$-test). No significant difference $(P>0.05$, one-way analysis of variance) was observed in the CA II immunostaining reactions between different cell-type categories, including spindle cell, epithelioid, or spindle/epithelioid variants. Mitotic index, which is an important prognostic indicator in GIST, showed statistically significant weak negative correlation to the CA II staining reactivity (Spearman $r=-0.22, P=0.0073$ ). CA II-negative GISTs seemed to have very high mitotic rates, accounting for this

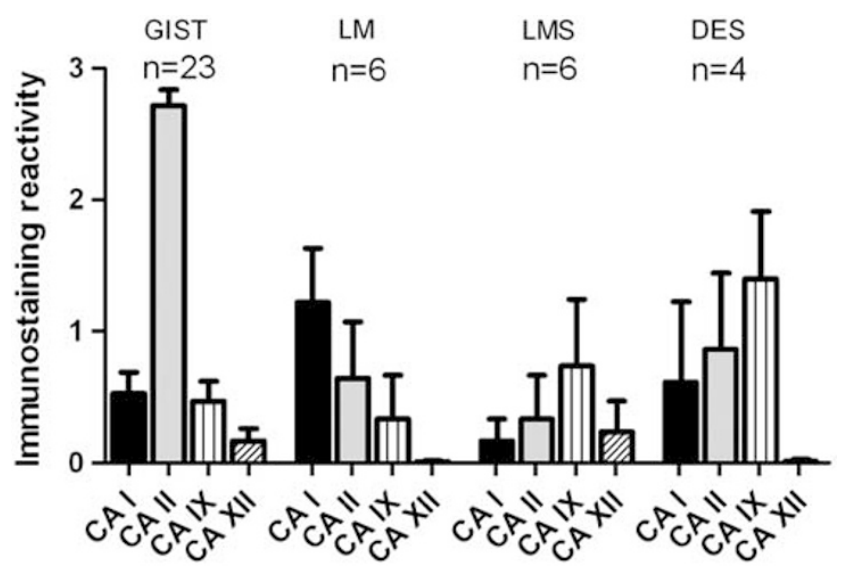

Figure 4 Distribution of mean ( $+/-$ s.e.m.) immunostaining reactivities for carbonic anhydrase I (CA I), CA II, CA IX, and CA XII in gastrointestinal stromal tumors (GISTs) and other mesenchymal tumors. The strongest immunoreactivities were observed for CA II in GISTs. LM=leiomyoma, LMS=leiomyosarcoma, $\mathrm{DES}=$ desmoid tumor.

negative correlation, but further studies are required because the series contained few CA II-negative or weakly stained GISTs. Another prognostic parameter, tumor size, showed no significant correlation to CA II immunoreactivity (Spearman $r=-0.03$, $P=0.6964)$.

\section{Prognostic Significance of CA II Expression}

Figure 5 shows disease-specific Kaplan-Meier plots for CA II immunostaining categories. The patients with strongly CA II-positive GISTs had a better prognosis. Log-rank (Mantel-Cox) test indicated that the curves were significantly different $(P<0.0001)$.

\section{CA II Expression in the Normal Gastrointestinal Canal}

In the stomach, small intestine and colon, Cajal cells were highlighted for CA II (Figure 6), whereas smooth muscle elements and neural plexuses were negative. In these segments of the gastrointestinal canal, especially the superficial mucosal elements were positive with both cytoplasmic and nuclear staining (data not shown). Gastric parietal cells and esophageal squamous epithelium were positive. Vascular endothelial cells were variably positive (Figure 6).

\section{Discussion}

This study shows that GISTs strongly express the CA II isozyme. Originally this enzyme was not considered an important target in cancer biology, because it is absent or only weakly expressed in most epithelial tumors. By contrast, various studies have included only the membrane-bound isoforms, CA 

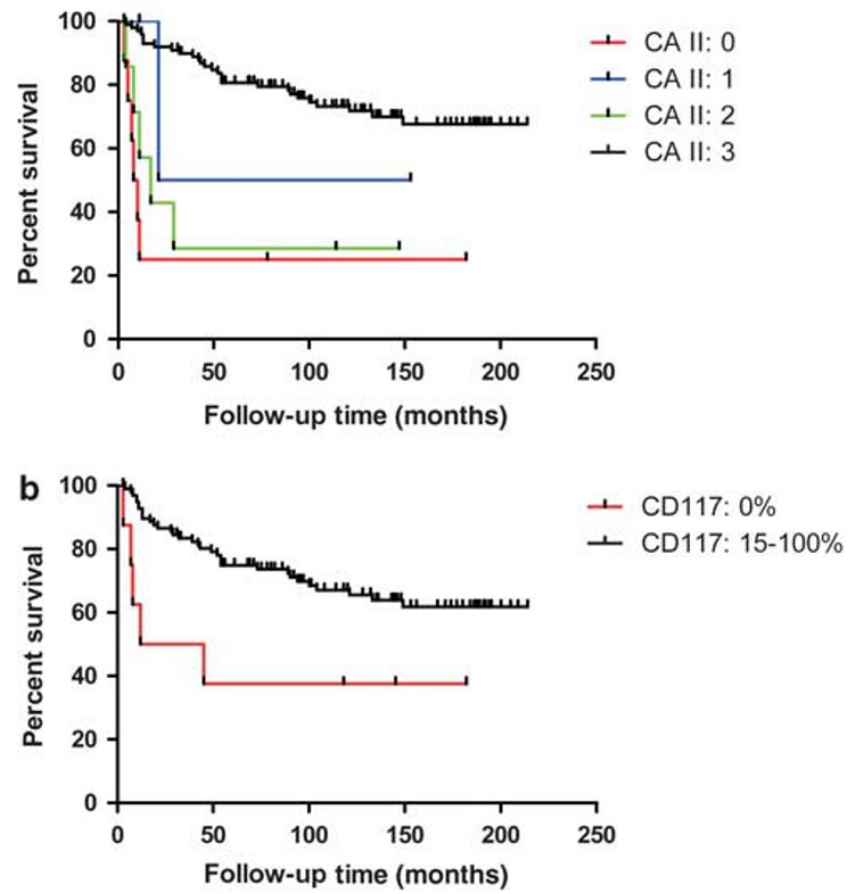

Figure 5 Disease-specific survival of gastrointestinal stromal tumor (GIST) patients categorized according to the carbonic anhydrase II (CA II) (a) or c-KIT (CD117) (b) immunostaining results. Positive reactions for KIT and strong staining for CA II indicated significantly better survival rates.

IX and XII, which are overexpressed in several types of cancers. ${ }^{22-25}$ There has been only scattered evidence that CA II is expressed to some extent in malignant cells such as leukemic blast cells $^{26}$ and colorectal, ${ }^{27,28}$ brain $^{27}$ and pancreatic ${ }^{29,30}$ cancers. A more recent study indicated that CA II expression is induced in neovascular endothelial cells of malignant melanoma and in esophageal, renal and lung cancers. $^{32}$ It was suggested that CA II associated with the neovascular endothelia could serve as a potential target for cancer therapy. It was also proposed that the presence of CA II in the endothelium could contribute to generation of autoantibody response that would, in turn, be a desired outcome in immune therapy of cancer.

In this study, we showed strong CA II immunoreactivity in GISTs, assessed with two independent antibodies and confirmed CA II expression by western blotting. Also, CA II transcript expression seems to be stronger in GIST than in many other sarcomas, as shown by gene expression profiles. ${ }^{28}$ GISTs have been thought to arise from progenitors of the interstitial cells of Cajal, which are found throughout the gastrointestinal tract from the esophagus to the internal anal sphincter. ${ }^{31,29}$ Cajal cells were the only mesenchymal elements positive for CA II in the normal gastrointestinal tract, indicating shared CA II-positive phenotype with GISTs, as has been previously observed for KIT and DOG1. ${ }^{30}$

Comparison of CA II expression in different tumor categories showed that the CA II immunoreactivity was markedly more common in GISTs than in the other mesenchymal tumors analyzed, suggesting that evaluation of CA II expression could be useful in the differential diagnosis of these entities, especially between GIST and leiomyosarcoma. Even though the specificity of CA II seemed to be quite good, small numbers of unrelated soft tissue tumors such as solitary fibrous tumors and malignant peripheral nerve sheath tumors were also occasionally positive. This result indicated that other markers must also be used, and the clinicopathologic context has to be considered. CA II seems to label GISTs independent of the site of origin or histologic type. Thus, epithelioid gastric GISTs, which are often less strongly KIT- and DOG1-positive, are strongly CA II-positive. Gastric spindle cell GISTs and small intestinal GISTs are also nearly uniformly positive. These findings indicate that CA II has some additional value in GIST diagnostics. Our statistical analysis also suggested that CA II expression may be associated with better prognosis, although this observation needs to be validated with a larger study cohort.

Our results showed that another cytosolic isozyme, CA I, is weakly expressed in GISTs and most cases remained completely negative. CA I was included in the immunohistochemical analysis, because $C A 1$ gene locus is located in close proximity to CA2 gene in the chromosome 8 (8q21.2). This result suggests that high expression of CA II in GISTs is not because of a gene amplification of the region that involves the cluster of genes encoding the cytosolic CAs. Importantly, the high CA II expression in GIST suggests that GIST cell lines may represent a valuable model in the study of the regulation and function of CA II in cancer cells, which has been a poorly understood research area to date. Possibly, CA II could have the same role in GIST as CA IX and XII in other tumors, ie, modulating intracellular $\mathrm{pH}$ balance and promoting extracellular acidification. Lower extracellular $\mathrm{pH}$ is generally considered a sign of aggressive phenotype of tumor cells and poorer prognosis, ${ }^{23}$ although in our GIST studies, higher CA II expression correlated with better prognosis. Therefore, it seems more likely that CA II expression might be associated with higher differentiation of GIST cells, and therefore, strong immunostaining correlates with favorable prognosis. The western blotting results showed that the CA II expression levels in GISTs are independent of KIT or PDGFRA mutations status.

In conclusion, our studies show CA II expression in GIST. The prognosis of GISTs with high CA II expression was better than that of GISTs with low or no expression, suggesting that CA II is both a diagnostic and prognostic biomarker for GIST. 

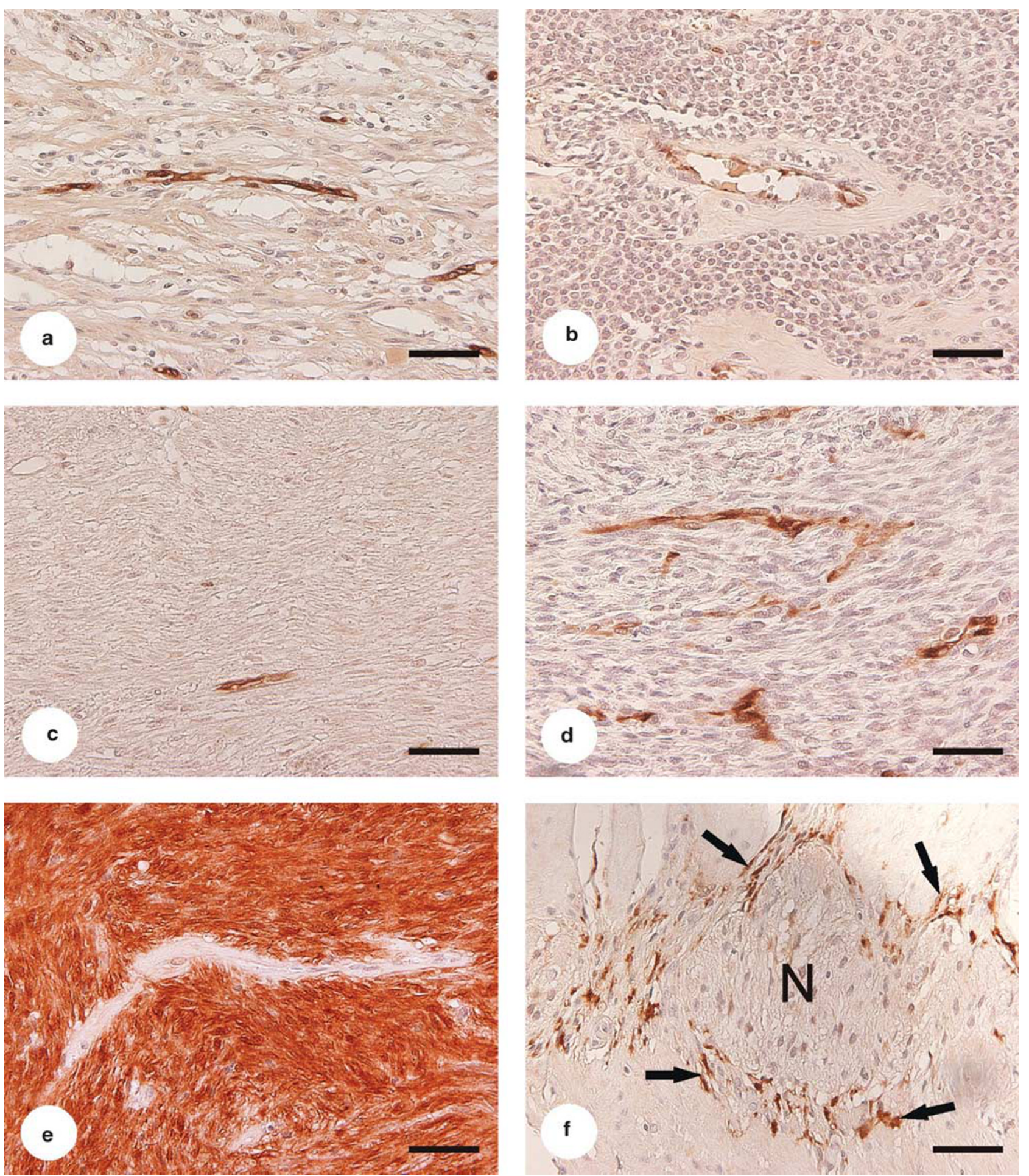

Figure 6 Carbonic anhydrase II (CA II) immunostaining in gastric schwannoma (a), gastric glomus tumor (b), esophageal leiomyoma (c), malignant peripheral nerve sheath tumor (d), gastrointestinal stromal tumor (GIST) (e), and normal smooth muscle (f). GIST shows strong staining for CA II. Capillary endothelium is positive in all other tumor specimens. Note the positive immunoreactions in Cajal cells (arrows in f). Neural myenteric plexus (N) is negative. Bars $=50 \mu \mathrm{m}$.

Further validation studies with other CA antibodies should be undertaken to characterize CA II expression in a larger cohort of GIST and other mesenchymal tumors of the gastrointestinal tract.

\section{Acknowledgement}

This work was supported by the following grants: DeZnIT European Union FP6 project (SP), a grant 
from Sigrid Juselius Foundation (SP), and GI SPORE 1P50CA127003-02 (JAF).

\section{Disclosure/conflict of interest}

The authors declare no conflict of interest.

\section{References}

1 Fletcher JA, Rubin BP. KIT mutations in GIST. Curr Opin Genet Dev 2007;17:3-7.

2 Lasota J, Miettinen M. Clinical significance of oncogenic KIT and PDGFRA mutations in gastrointestinal stromal tumours. Histopathology 2008;53:245-266.

3 Miettinen M, Lasota J. Gastrointestinal stromal tumors: review on morphology, molecular pathology, prognosis, and differential diagnosis. Arch Pathol Lab Med 2006;130:1466-1478.

4 Kindblom LG, Remotti HE, Aldenborg F, et al. Gastrointestinal pacemaker cell tumor (GIPACT): gastrointestinal stromal tumors show phenotypic characteristics of the interstitial cells of Cajal. Am J Pathol 1998;152:1259-1269.

5 DeMatteo RP, Lewis JJ, Leung D, et al. Two hundred gastrointestinal stromal tumors: recurrence patterns and prognostic factors for survival. Ann Surg 2000; 231:51-58.

6 Sarlomo-Rikala M, Kovatich AJ, Barusevicius A, et al. CD117: a sensitive marker for gastrointestinal stromal tumors that is more specific than CD34. Mod Pathol 1998;11:728-734.

7 Katz SC, DeMatteo RP. Gastrointestinal stromal tumors and leiomyosarcomas. J Surg Oncol 2008;97:350-359.

8 Joensuu H. Gastrointestinal stromal tumor (GIST). Ann Oncol 2006;17(Suppl 10):x280-x286.

9 Miettinen MM, Sarlomo-Rikala M, Kovatich AJ, et al. Calponin and h-caldesmon in soft tissue tumors: consistent h-caldesmon immunoreactivity in gastrointestinal stromal tumors indicates traits of smooth muscle differentiation. Mod Pathol 1999;12:756-762.

10 Tazawa K, Tsukada K, Makuuchi $\mathrm{H}$, et al. An immunohistochemical and clinicopathological study of gastrointestinal stromal tumors. Pathol Int 1999;49: 786-798.

11 Tsujimura T, Makiishi-Shimobayashi C, Lundkvist J, et al. Expression of the intermediate filament nestin in gastrointestinal stromal tumors and interstitial cells of Cajal. Am J Pathol 2001;158:817-823.

12 Blay P, Astudillo A, Buesa JM, et al. Protein kinase C theta is highly expressed in gastrointestinal stromal tumors but not in other mesenchymal neoplasias. Clin Cancer Res 2004;10:4089-4095.

13 Duensing A, Joseph NE, Medeiros F, et al. Protein kinase $\mathrm{C}$ theta (PKCtheta) expression and constitutive activation in gastrointestinal stromal tumors (GISTs). Cancer Res 2004;64:5127-5131.

14 Espinosa I, Lee CH, Kim MK, et al. A novel monoclonal antibody against DOG1 is a sensitive and specific marker for gastrointestinal stromal tumors. Am J Surg Pathol 2008;32:210-218.

15 West RB, Corless CL, Chen X, et al. The novel marker, DOG1, is expressed ubiquitously in gastrointestinal stromal tumors irrespective of KIT or PDGFRA mutation status. Am J Pathol 2004;165:107-113.

16 Chiche J, Ilc K, Laferriere J, et al. Hypoxia-inducible carbonic anhydrase IX and XII promote tumor cell growth by counteracting acidosis through the regulation of the intracellular $\mathrm{pH}$. Cancer Res 2009; 69:358-368.

17 Parkkila AK, Parkkila S, Juvonen T, et al. Carbonic anhydrase isoenzymes II and I are present in the zona glomerulosa cells of the human adrenal gland. Histochemistry 1993;99:37-41.

18 Kivela A, Parkkila S, Saarnio J, et al. Expression of a novel transmembrane carbonic anhydrase isozyme XII in normal human gut and colorectal tumors. Am J Pathol 2000;156:577-584.

19 Karhumaa P, Parkkila S, Tureci O, et al. Identification of carbonic anhydrase XII as the membrane isozyme expressed in the normal human endometrial epithelium. Mol Hum Reprod 2000;6:68-74.

20 Pastorekova S, Zavadova Z, Kostal M, et al. A novel quasi-viral agent, MaTu, is a two-component system. Virology 1992;187:620-626.

21 Tuveson DA, Willis NA, Jacks T, et al. STI571 inactivation of the gastrointestinal stromal tumor c-KIT oncoprotein: biological and clinical implications. Oncogene 2001;20:5054-5058.

22 Parkkila S, Rajaniemi H, Parkkila AK, et al. Carbonic anhydrase inhibitor suppresses invasion of renal cancer cells in vitro. Proc Natl Acad Sci USA 2000; 97:2220-2224.

23 Pastorekova S, Parkkila S, Pastorek J, et al. Carbonic anhydrases: current state of the art, therapeutic applications and future prospects. J Enzyme Inhib Med Chem 2004;19:199-229.

24 Pastorekova S, Zavada J. Carbonic anhydrase IX (CA IX) as a potential target for cancer therapy. Cancer Ther 2004;2:245-262.

25 Ivanov S, Liao SY, Ivanova A, et al. Expression of hypoxia-inducible cell-surface transmembrane carbonic anhydrases in human cancer. Am J Pathol 2001; 158:905-919.

26 Leppilampi M, Koistinen P, Savolainen ER, et al. The expression of carbonic anhydrase II in hematological malignancies. Clin Cancer Res 2002;8:2240-2245.

27 Parkkila AK, Herva R, Parkkila S, et al. Immunohistochemical demonstration of human carbonic anhydrase isoenzyme II in brain tumours. Histochem J 1995;27: 974-982.

28 Nielsen TO, West RB, Linn SC, et al. Molecular characterisation of soft tissue tumours: a gene expression study. Lancet 2002;359:1301-1307.

29 Hagger R, Gharaie S, Finlayson C, et al. Distribution of the interstitial cells of Cajal in the human anorectum. J Auton Nerv Syst 1998;73:75-79.

30 Miettinen M, Wang ZF, Lasota J. DOG1 antibody in the differential diagnosis of gastrointestinal stromal tumors: a study of 1840 cases. Am J Surg Pathol 2009;33: 1401-1408.

31 Daniel EE, Posey-Daniel V. Neuromuscular structures in opossum esophagus: role of interstitial cells of Cajal. Am J Physiol 1984;246:G305-G315.

32 Yoshiura K, Nakaoka T, Nishishita T, et al. Carbonic anhydrase II is a tumor vessel endothelium-associated antigen targeted by dendritic cell therapy. Clin Cancer Res 2005;11:8201-8207. 